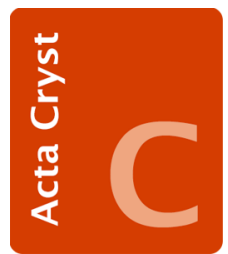

STRUCTURAL

CHEMISTRY

Volume 74 (2018)

Supporting information for article:

Exploring a solvated dimer of Gefitinib: a quantitative analysis

This article is dedicated to Professor K. S. Viswanathan in celebration of his 65 th birthday.

Deekshi Angira, Althaf Shaik, Sivapriya Kirubakaran and Vijay Thiruvenkatam 


\section{Fingerprint plot analysis}

Fingerprint plots that elucidate the contribution of various contacts present in the system were generated using the same software. Figs. 8 (see paper) and S1 (below) clearly signify that the three forms do not differ much with respect to the type of contacts that dominate in each. The few notable differences in the percentage contribution of contacts are for the C...H (12.6\%) and O...H (11.7\%) contacts in the solvated form, which is a little greated than that in form I (C...H 11.7\% and O...H 9.2\%) and form II (C...H 12.9\% and O...H 10\%), and the N...H contact, which is less in the solvated form (6\%) compared to forms I (7\%) and II (7.1\%). The contributions are detailled in Fig. 8 (see paper).
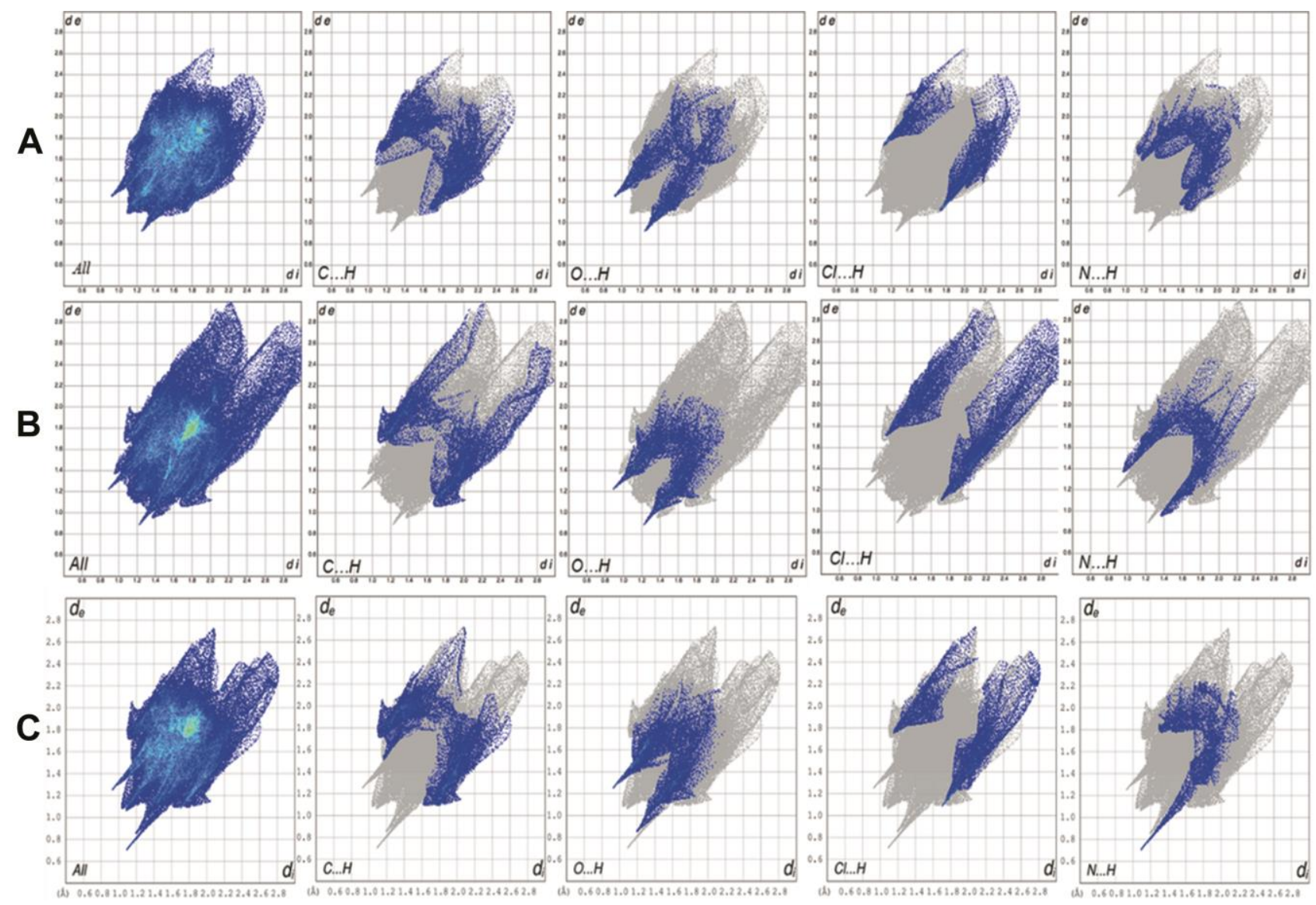

Fig. S1. A comparison of two-dimensional fingerprint plots of the Gefitinib polymorphs, where row $\mathbf{A}$ is Form I, row $\mathbf{B}$ is Form II and row $\mathbf{C}$ is the solvated dimer of Gefitinib (molecule $A$ ). 